\title{
Cardiac Resynchronization Guided by Ultra-High-Frequency ECG Maps
}

\author{
Pavel Jurak ${ }^{1}$, Magdalena Matejkova ${ }^{2}$, Josef Halamek ${ }^{1}$, Filip Plesinger ${ }^{1}$, Ivo Viscor ${ }^{1}$, Vlastimil \\ Vondra $^{1}$, Jolana Lipoldova ${ }^{2}$, Miroslav Novak ${ }^{2}$, Radovan Smisek ${ }^{1}$, Pavel Leinveber ${ }^{2}$ \\ ${ }^{1}$ The Czech Academy of Sciences, Institute of Scientific Instruments, Brno, CZ \\ ${ }^{2}$ International Clinical Research Center, St. Anne’s University Hospital, Brno, CZ
}

\begin{abstract}
Here we present a technique based on 14-lead ultrahigh-frequency electrocardiogram (UHF-ECG, $5 \mathrm{kHz}$ ) able to measure the immediate effect of cardiac resynchronization therapy (CRT) settings on electrical ventricular dyssynchrony.

Resting UHF-ECGs were sequentially recorded during different CRT settings: CRT OFF, CRT ON, VV delays 0 , -20, -40 ms, and SMART sensing (Boston Scientific) in 33 patients. Electrical depolarization maps were computed from signal-averaged UHF-ECGs in the frequency band 150-1000 Hz. The horizontal axis of each map indicates time and the vertical axis indicates the activation location (V1-V8 lead). The red areas show the location-time of the maximal volume of simultaneously depolarized myocardial cells.

The optimal CRT setting is characterized by a narrow and vertical activation pattern. Optimal CRT setting selected by a physician from electrical depolarization maps corresponded to the minimal mechanical intraventricular delay obtained from echocardiography in $61 \%$ of patients; in $27 \%$ of patients the difference was within a 10 ms interval.

UHF-ECG depolarization maps represent a useful tool for the selection and setting of optimal cardiac resynchronization therapy.
\end{abstract}

\section{Introduction}

Cardiac resynchronization therapy (CRT) aims to eliminate ventricular dyssynchrony. However, approximately 30-50 percent of patients are still not appropriately treated. New stimulation technologies allow successful CRT even in patients who do not meet general criteria [1]. On the other hand, however, patients who meet these criteria may not respond positively. CRT treatment requires a comprehensive approach that links all stages throughout the CRT procedure; not only CRT responsibility predicting, but also technology selection and the settings and optimization of CRT parameters.

It is inaccurate to select the patient for CRT treatment according to established electrocardiographic (ECG) parameters. If the patient has a QRS duration above 150 ms, clear LBBB morphology or high QRS area [2], this is not a guarantee that he will respond positively. If the ECG parameters are above or below predefined limits, a higher or lower probability of positive response is expected. This probability may not be the same for different patient groups and different pacing technologies and criteria. Thus, the extensive large cohort trials provide results that do not advise the physician as to how to resynchronize a particular patient with an individual approach. Additionally, the limited sensitivity and specificity of ECG parameters require years for verification. This critically slows down the implementation of new stimulation techniques.

Here we present a new electrocardiographic method based on 14-lead ultra-high-frequency ECG (UHF-ECG) able to measure the immediate effect of CRT settings on electrical ventricular resynchronization.

\section{Methods}

\subsection{Data recording}

A 14-lead ECG was collected at $5 \mathrm{kHz}$ with a dynamic range of 26 bits and a $2 \mathrm{kHz}$ passband (VDI monitor, ISI, Cardion, FNUSA Brno, CZ), Figure 1. Measurements were taken at the International Clinical Research Center at St. Anne's University Hospital, Brno, Czech Republic.

Resting 1-3 minute UHF-ECGs were sequentially recorded during different Biventricular CRT settings: baseline (CRT OFF), CRT ON, ventriculo-ventricular (VV) delay 0, -20, -40 ms, and SMART sensing (Boston Scientific automatically optimized atrioventricular and 
intraventricular delay) in 34 patients (24 men and 10 women, age $65.0 \pm 10.1$ ).

All subjects gave their informed consent to the investigation. The study was approved by the Ethics Committee of St. Anne's University Hospital, Brno.

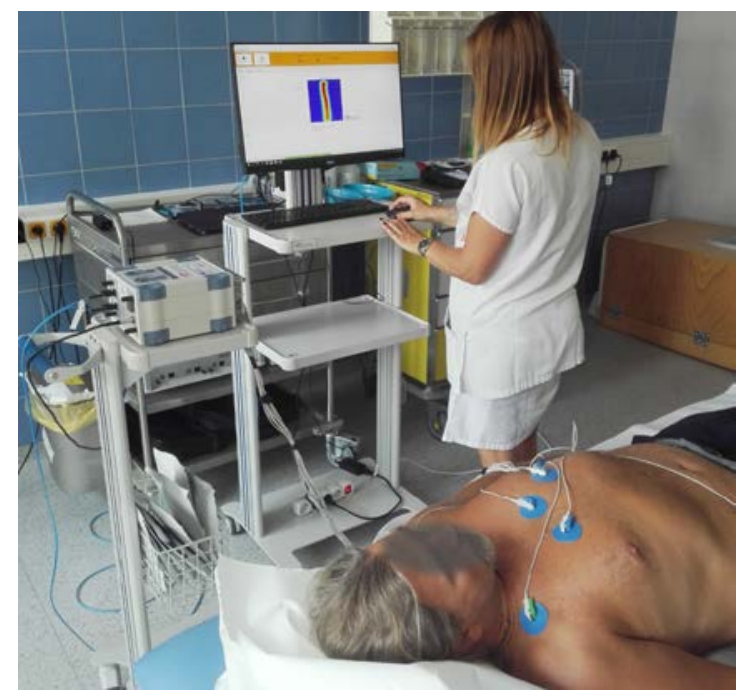

Figure 1. 14-lead UHF-ECG VDI (Ventricular Dyssynchrony Imaging) monitor for real-time monitoring of depolarization pattern. The VDI monitor can provide the first results within 20 seconds. Installation at St. Anne’s University Hospital, Brno, Czech Republic, 2019.

\subsection{Processing}

Electrical depolarization maps (Figure 2B) were computed from signal-averaged UHF-ECGs in the frequency band 150-1000 Hz. The horizontal axis of each map indicates time and the vertical axis indicates the activation location (V1-V8 leads). The red areas show the location-time of the maximal volume of simultaneously depolarized myocardial cells. The optimal CRT setting is characterized by a narrow and vertical activation pattern (Figure 2b, bottom map, biventricular pacing). Narrowing means faster depolarization conduction, and a straight vertical pattern means the elimination of ventricular dyssynchrony. The electrical dyssynchrony (e-DYS) was estimated as the time delay between the first and last activation, Figure 2B.

The arrows in Figure 2B indicate a slow conduction region close to the V2 and V3 leads. In this area, the depolarization propagation slow-down and dyssynchrony increases.
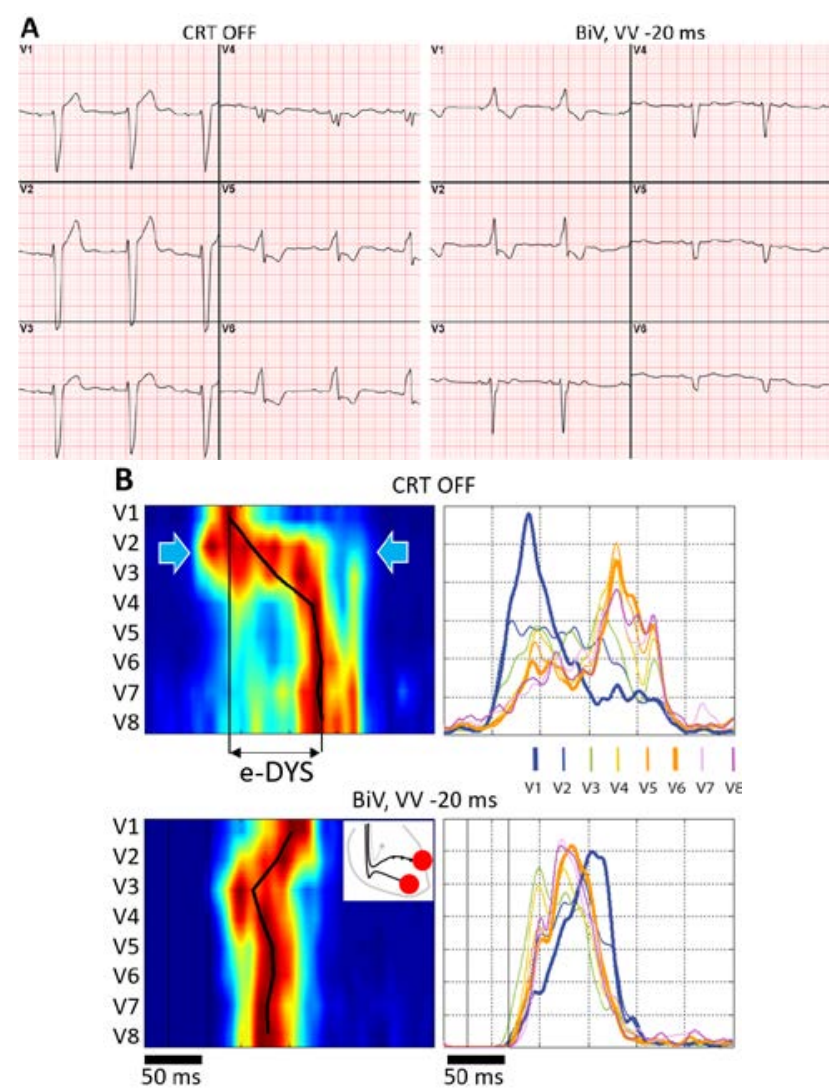

Figure 2. Determination of resynchronization effect by electrical depolarization maps; during CRT OFF and CRT ON (biventricular stimulation). Left bundle branch block (LBBB) patient.

A - precordial leads V1-V6, B - left panel: electrical depolarization maps, right panel: depolarization distribution in V1-V8 leads - amplitude envelopes.
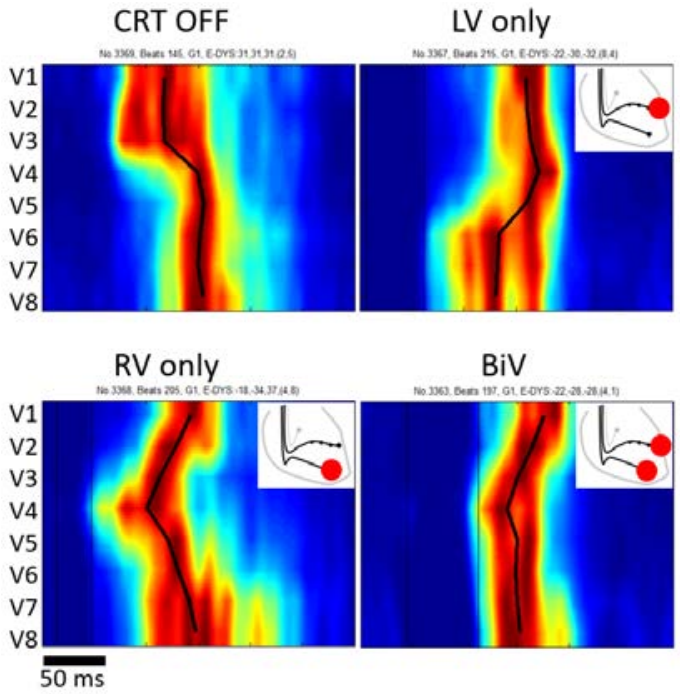

Figure 3. Electrical depolarization maps, LBBB patient 
1 - CRT OFF, 2 - left ventricle pacing, 3 - right ventricle pacing, 4 - biventricular pacing.

Figure 2 shows the elimination of dyssynchrony during biventricular stimulation. Dyssynchrony reduction is identifiable from the depolarization maps much more clearly and easily than from a conventional ECG.

Figure 3 shows examples of how the depolarization maps change with different pacing site: stimulation in the right ventricular apex (RV only), coronary sinus (LV only) and finally biventricular pacing. It is evident that it is important to consider not only dyssynchrony, but also the whole depolarization activation pattern.

Figure 4 shows different VV delays $0,-20,-40$ ms. In this case, dyssynchrony gradually decreases from $65 \mathrm{~ms}$ (OFF) to 36, 15, and $11 \mathrm{~ms}$. The optimal VV delay setting based on the depolarization map assessment is $\mathrm{VV}=-40$ ms.

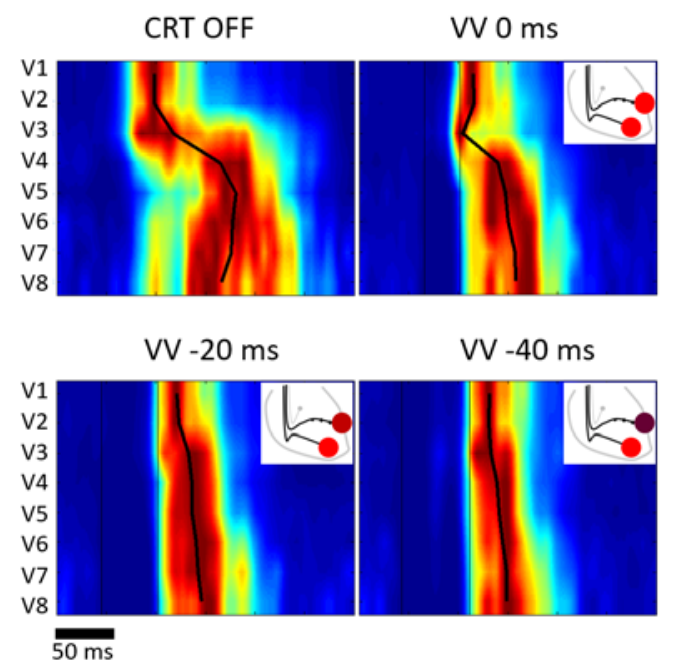

Figure 4. Electrical depolarization maps during different left ventricle pre-excitation (ventriculoventricular VV delay) during biventricular pacing. VV=40 ms was chosen as the optimal setting.

\section{Results}

The optimal CRT setting was selected by a physician from electrical activation maps for different VV delays. The mechanical intraventricular delay obtained from echocardiography was measured in all patients during CRT OFF, previously selected optimal CRT (assessed by UHF-ECG) and SMART CRT. The mechanical intraventricular delays were compared with electrical dyssynchrony. In $61 \%$ of patients, minimal mechanical intraventricular delay corresponded to the optimal VV delay setting. In $27 \%$ of patients, the positive or negative effect of stimulation cannot be conclusively assessed. In these patients, the e-DYS difference between optimal and
SMART setting was lower than a $10 \mathrm{~ms}$ interval. In $12 \%$ of patients, the minimal mechanical intraventricular delay corresponds to the SMART setting, Figure 5.

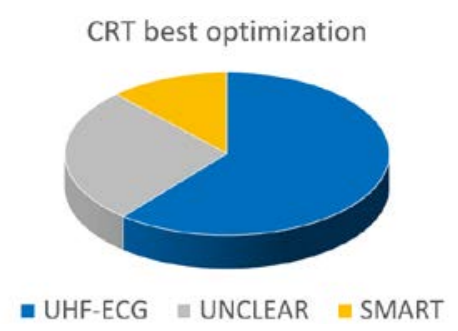

Figure 5. UHF-ECG optimization is the best solution in $61 \%$ of patients. The SMART CRT setting is beneficial in only $12 \%$ of patients. In $27 \%$ of the patients, the resynchronization effect is unclear, and it was not possible to reliably assess the positive or negative settings.

\section{Discussion}

This study aims to introduce a new technique for measuring the properties of ventricular depolarization propagation during different CRT settings. The UHFECG method was first introduced in [3], and clinical benefit was tested on a large MADIT-CRT $1 \mathrm{kHz}$ Holter database [4] in the high-frequency range up to $350 \mathrm{~Hz}$. Recently the study [5] has compared ventricular dyssynchrony with other ECG parameters. Ventricular dyssynchrony estimated from high-frequency ECG has been confirmed as a parameter that best predicts positive CRT outcome in left bundle branch block patients. Unfortunately, so far there is no large-cohort trial and statistics on data with ultra-high-frequency ECGs.

The aim of this study is to introduce and discuss the importance of a different approach to CRT. A significant improvement in positive outcome can be expected when it is possible to assess interactively the immediate effect of resynchronization at the time of pacemaker implantation. Such an approach could allow selection of resynchronization technology (biventricular pacing, His bundle pacing) and adjustment of pacing sites, thresholds and delays.

The 12-lead ECG is the primary source of information to optimize pacing settings during implantation and to optimize CRT parameters during the follow-up period. The main criteria are QRS morphology and duration. However, assessing small changes in these parameters during implantation is complicated and inaccurate. This leads to the fact that the pacemaker implantation and post-implantation setting may not be optimal.

Electrical depolarization maps provide deterministic information about depolarization properties during pacing without human intervention. Both the graphical 
representation and numerical value (e-DYS) indicate the changes of ventricular electrical dyssynchrony during different settings of CRT. Therefore, UHF-ECG provides information that is essential for making immediate decisions about CRT settings.

To partially confirm these assumptions, an example of the utilization of the UHF-ECG methodology during optimization of post-implantation CRT settings is introduced. It has been shown in a relatively small set of patients that UHF-ECG allows optimization of VV delays to achieve better resynchronization results than the recommended SMART solution. More information on this study can be found at [6].

\section{Conclusion}

UHF-ECG electrical depolarization maps provide a deterministic and straightforward insight into the ventricular depolarization activation pattern. Simultaneous comparison of multiple maps for different CRT settings can be useful for the selection of optimal pacemaker configuration.

\section{Acknowledgments}

The research was supported by the Czech Science Foundation, project GA17-13830S, by the Ministry of Education, Youth and Sports of the Czech Republic (project LO1212 and LQ1605) and by the Czech Academy of Sciences (project RVO:68081731).

\section{References}

[1] M. Brignole et al., "Guidelines on cardiac pacing and cardiac resynchronization therapy”, Acta Cardiol. vol. 69, no. 1, pp. 52-53, 2014, doi:10.2143/AC.69.1.3011345.

[2] UC. Nguyên, S. Claridge, K. Vernooy, EB. Engels, R. Razavi, CA. Rinaldi et al., "Relationship between vectorcardiographic QRSarea, myocardial scar quantification, and response to cardiac resynchronization therapy”, J Electrocardiol, vol. 51, pp. 457-63, 2018 doi:10.1016/j.jelectrocard.2018.01.009.

[3] P. Jurak, J. Halamek, J. Meluzin, F. Plesinger, T. Postranecka, J. Lipoldova, M. Novak, V. Vondra, I. Viscor, L. Soukup, P. Klimes, P. Vesely, J. Sumbera, K. Zeman, RS. Asirvatham, J. Tri, SJ. Asirvatham, P. Leinveber, "Ventricular dyssynchrony assessment using ultra-high frequency ECG technique”, J Interv Card Electrophysiol, vol. 49, no. 3, pp. 245-254, 2017, doi:10.1007/s10840-0170268-0.

[4] F. Plesinger, P. Jurak, J. Halamek, P. Nejedly, P. Leinveber, I. Viscor, V. Vondra, S. McNitt, B. Polonsky, AJ. Moss, W. Zareba, JP. Couderc, "Ventricular electrical delay measured from body surface ECGs is associated with cardiac resynchronization therapy response in left bundle branch block patients from the MADIT-CRT trial (Multicenter automatic defibrillator implantation-cardiac resynchronization therapy)", Circ Arrhythmia Electrophysiol, vol. 11, no. 5, 2018 doi:10.1161/circep.117.005719.

[5] J. Halamek, P. Leinveber, I. Viscor, R. Smisek, F. Plesinger, V. Vondra, P. Jurak, “The relationship between ECG predictors of cardiac resynchronization therapy benefit”, PLoS One, vol. 14, no. 5, pp. 1-10, 2019, doi:10.1371/journal.pone.0217097.

[6] M. Matejkova, J. Lipoldova, P. Leinveber, J. Halamek, P. Jurak, F. Plesinger, A. Nagy, M. Novak, "Optimized CRT stimulation based on ultra-high-frequency QRS analysis”, Computing in Cardiology, 2019.

Address for correspondence:

Pavel Jurak

Institute of Scientific Instruments of the CAS

Kralovopolska 147, 61264 Brno

Czech Republic

jurak@isibrno.cz 